\title{
Postsurgical lobar torsion in an unusual setting
}

Miyako Hiramatsu, MD, Kiyomi Shimoda, MD, Naoya Katsuragi, MD, and Yuji Shiraishi, MD, Tokyo, Japan

Postsurgical lobar torsion is a rare complication. It can occur as a result of the intraoperative manipulation of intrathoracic organs and is considered to be established before reexpansion of the lung after the one lung ventilation. We experienced a case with postsurgical lobar torsion of a different underlying etiology in a patient who had undergone a right upper lobectomy for the treatment of a giant bulla that occupied almost the entire right hemithorax. The residual middle and lower lobes became severely atelectatic 2 days after the operation, when the patient started to move around with a chest tube in place without suction. This was probably caused by the increased elastic recoil of the residual lobes as a result of long-term compression atelectasis before surgery. Lobar torsion was then established when the lobes were reexpanded with the additional drainage with suction. Postsurgical lobar torsion in such an unusual setting has never been reported previously.

\section{CLINICAL SUMMARY}

A 35-year-old man was seen at our institution with persistent cough. His serial chest radiographs through a few years showed an enlarging giant bulla that finally occupied his entire right hemithorax and compressed the normal lung structure. We decided to perform a right upper lobectomy instead of a bullectomy because the upper lobe was full of innumerable medium sized bullae. Because the inferior pulmonary ligament was left undivided, the residual lobes were not fixed over the complete interlobar fissure. The chest radiograph taken before extubation confirmed that there was no malpositioning of the lung under positive airway pressure. Suction was first applied to the chest tube, and then the tube was placed under a water seal at the ward.

The early postoperative course was uneventful. When the patient started to move around on postoperative day 2, however, he reported postural tachycardia, and serous-bloody chest tube drainage increased from a total of 10 to 900 $\mathrm{mL}$ in the course of 24 hours. A chest radiograph taken the same day showed an obvious volume loss of the right lung (Figure 1). Urgent bronchoscopy revealed a slightly stenotic intermediate bronchus without mucous plugging.

\footnotetext{
From the Section of Chest Surgery, Fukujuji Hospital, Tokyo, Japan.

Disclosures: Authors have nothing to disclose with regard to commercial support.

Received for publication June 5, 2014; revisions received Sept 17, 2014; accepted for publication Oct 6, 2014; available ahead of print Nov 5, 2014

Address for reprints: Miyako Hiramatsu, MD, Section of Chest Surgery, Fukujuji

Hospital, 3-1-24, Matsuyama, Kiyose-shi, Tokyo 204-8522, Japan (E-mail:

mykhrmt@a07.itscom.net).

J Thorac Cardiovasc Surg 2015;149:e1-2

$0022-5223 / \$ 36.00$

Copyright (C) 2015 by The American Association for Thoracic Surgery

http://dx.doi.org/10.1016/j.jtcvs.2014.10.053
}

After the second drainage tube was inserted and connected to suction, the lung partially reexpanded (Figure 2). A computed tomographic scan was performed on postoperative day 6 to evaluate the unimproved atelectasis; this scan revealed lobar torsion of the middle and lower lobes.

The patient underwent urgent reoperation. The diaphragmatic surface of the partially aerated lower lobe faced upward. The middle lobe, whose hemorrhagic infarction was obvious from its dark and consolidated appearance, was located in the posterior costophrenic space. By exploring the hilum, we found that the lobes were twisted together counterclockwise by $180^{\circ}$. After we performed a middle lobectomy, the patient remained on a ventilator for 5 days in the intensive care unit to treat reexpansion pulmonary edema. He was discharged 29 days after rethoracotomy. The resected specimen showed increased elastic fiber within the compressed nonbullous area, suggesting that its inability to remain inflated without suction was related to its increased elastic recoil.

\section{DISCUSSION}

Postsurgical lobar torsion is a rare but potentially lethal postoperative complication that is induced by the

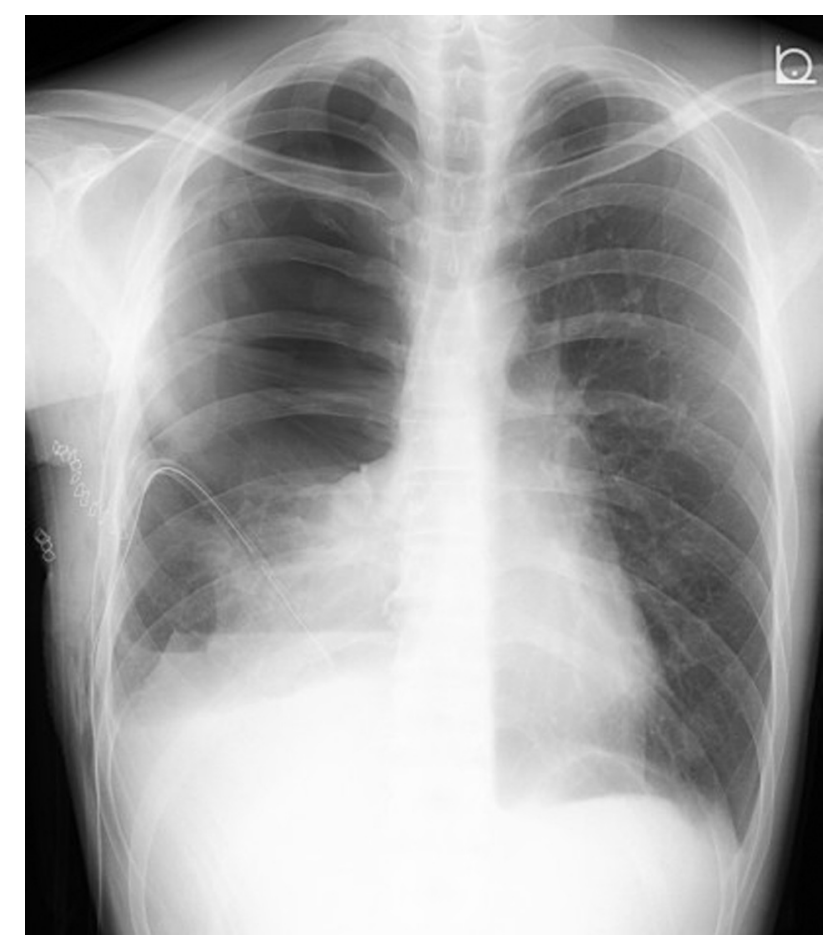

FIGURE 1. Chest radiograph of severely deflated middle and lower lobes 2 days after the initial operation. 


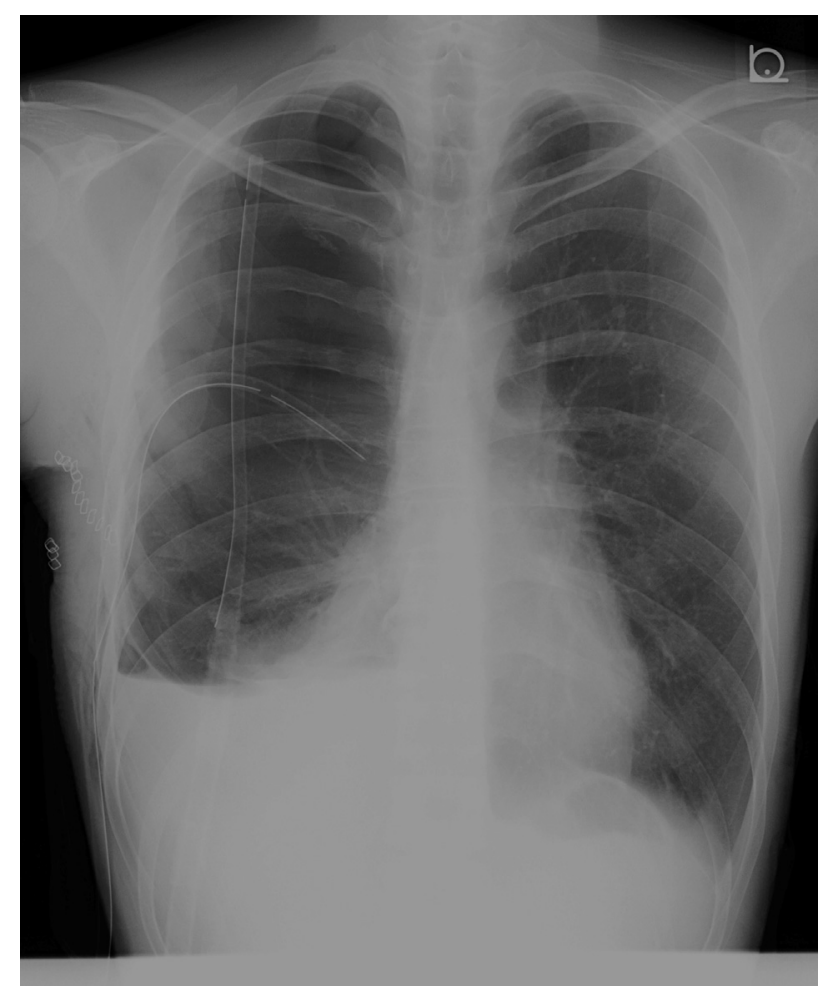

FIGURE 2. Chest radiograph demonstrating that after the additional drainage with suction, the lung became partially reinflated. Note the abnormal vasculature of the hilum.

malpositioning of the lung at the end of thoracic surgery. ${ }^{1,2}$ It is considered that complete deflation of the lung with a double-lumen tube makes torsion possible. ${ }^{3,4}$ Other reported causes of lobar torsion include trauma, pneumonia, and most commonly atelectasis with an underlying etiology such as spontaneous or iatrogenic pneumothorax, massive pleural effusion, or airway obstruction. ${ }^{5}$ In our case, the residual lobes were in the proper position at the end of the right upper lobectomy. Although these lobes hardly remained inflated by postoperative day 2 , the patient became sick. Bronchoscopy and additional drainage could not demonstrate the cause of the lobar atelectasis. The altered vasculature on chest radiography after the tube insertion suggests that the torsion may have occurred while the lobes were being reexpanded by this additional suction. This unusual cause and process made the diagnosis of lobar torsion difficult and delayed.

In the case of postoperative lobar atelectasis, rigorous radiologic and bronchoscopic evaluation to identify the underlying etiology and its appropriate management is crucial. This case also tells us that lobar torsion can occur during the reexpansion of collapsed residual lobes. To avoid this complication, the residual lobe should be fixed, as is widely recommended. ${ }^{1,4}$ In addition, suction should be applied to the chest tube for long enough to keep the lung inflated after long-term compression.

\section{References}

1. Cable DG, Deschamps C, Allen MS, Miller DL, Nichols FC, Trastek VF, et al. Lobar torsion after pulmonary resection: presentation and outcome. J Thorac Cardiovasc Surg. 2001;122:1091-3.

2. Felson B. Lung torsion: radiographic findings in nine cases. Radiology. 1987;162: 631-8.

3. Oddi MA, Taugott RC, Will RJ, Simmons RA, Treasure RL, Schuchmann GF. Unrecognized intraoperative torsion of the lung. Surgery. 1981;89:390-3.

4. Larsson S, Lepore V, Dernevik L, Nilsson F, Selin K. Torsion of a lung lobe: diagnosis and treatment. Thorac Cardiovasc Surg. 1988;36:281-3.

5. Moser ES Jr, Proto AV. Lung torsion: case report and literature review. Radiology. $1987 ; 162: 639-43$.

\title{
Novel sequential bridge to lung transplant in an awake patient
}

\author{
Nikhil P. Patil, MS, MRCS (Eng), MCh (CTVS), ${ }^{\mathrm{a}}$ Aron F. Popov, MD, PhD, ${ }^{\mathrm{a}}$ \\ Nicholas J. Lees, MBChB, FRCA, FFICM, ${ }^{b}$ and André R. Simon, MD, PhD, ${ }^{a}$ London, United Kingdom
}

\footnotetext{
From the Department of Cardiothoracic Transplantation and Mechanical Support, ${ }^{2}$ and the Department of Anaesthesia and Critical Care, ${ }^{\mathrm{b}}$ Royal Brompton and Harefield NHS Foundation Trust, Harefield Hospital, London, UK.

Disclosures: Authors have nothing to disclose with regard to commercial support.

Received for publication July 3, 2014; revisions received Sept 4, 2014; accepted for publication Oct 5, 2014; available ahead of print Nov 7, 2014

Address for reprints: Nikhil P. Patil, MS, MRCS (Eng), MCh (CTVS), Department of Cardiothoracic Transplantation and Mechanical Circulatory Support, Harefield Hospital, London, United Kingdom (E-mail: n.patil@rbht.nhs.uk).

J Thorac Cardiovasc Surg 2015;149:e2-4

$0022-5223 / \$ 36.00$

Copyright (c) 2015 by The American Association for Thoracic Surgery

http://dx.doi.org/10.1016/j.jtcvs.2014.10.031
}

Patients awaiting lung transplant (LTx) may have acute deterioration, but preoperative invasive mechanical ventilation (IMV) is undesirable because it increases mortality associated with LTx. ${ }^{1}$ We describe our experience of bridging to LTx while avoiding IMV by using the Hemolung (ALungTechnologies, Pittsburgh, Pa), a novel extracorporeal carbon dioxide removal $\left(\mathrm{ECCO}_{2} \mathrm{R}\right)$ device for partial respiratory support, with subsequent extracorporeal membrane oxygenation (ECMO) through in situ Hemolung cannulas for escalated support. 